\title{
ON SPLITTING AUGMENTATION IDEALS
}

\author{
WARREN DICKS
}

\begin{abstract}
Let $G$ be a group, $H$ a subgroup of $G$ and $R$ an associative ring. Write $\omega(R G)$ for the augmentation ideal of the group ring $R G$, and $\omega(R H) G$ for the right ideal of $R G$ generated by $\omega(R H)$. For $G$ finitely generated over $H$ we characterize, in terms of the Bass-Serre theory of groups acting on trees, the situation where $\omega(R H) G$ is an $R G$-summand of $\omega(R G)$.
\end{abstract}

Let $G$ be a group, $H$ a subgroup of $G$ and $R$ an associative ring with $1 \neq 0$.

We write $R G$ for the group ring, $\omega(R G)$ for its augmentation ideal, and $\omega(R H) G$ for the right ideal of $R G$ generated by $\omega(R H)$.

The situation where $\omega(R H) G$ is an $R G$-summand of $\omega(R G)$ has been considered by various authors [1], [4], [5], [6], and the purpose of this paper is to give the following characterization in terms of the Bass-Serre theory of groups acting on trees. (We write $R^{-1}$ for the set of positive integers invertible in $R$; for other terminology we refer to [2].)

THEOREM A. If $G$ is finitely generated over $H$ then the following are equivalent:

(1) $\omega(R H) G$ is an $R G$-summand of $\omega(R G)$.

(2) $G$ can be expressed as the fundamental group of a graph of groups $(\mathcal{G}, Y)$

with $Y$ finite,

with finite edge groups,

with $H$ as one of the vertex groups,

such that for each infinite vertex group $\mathcal{G}(v)$ distinct from $H,\left|H \cap \mathcal{G}(v)^{g}\right| \in R^{-1}$ for all $g \in G$,

and with $\left|H \cap H^{g}\right| \in R^{-1}$ for all $g \notin H$.

(3) $G$ acts on a tree $X$

with $X / G$ finite,

with finite edge stabilizers,

with a vertex $v_{0}$ having stabilizer precisely $H$,

such that for each $v \neq v_{0}$ having star(v) infinite $\left|H \cap G_{v}\right| \in R^{-1}$,

and with $\left|H \cap H^{g}\right| \in R^{-1}$ for all $g \notin H$.

$\$ 1$ below is devoted to proving this. The equivalence of (2) and (3) is immediate from the Bass-Serre Structure Theorem; the equivalence of (1) and (2) will be derived using a result proved in [3] and formulated in [2, p. 81] as follows.

Received by the editors November 11, 1980.

1980 Mathematics Subject Classification. Primary 16A27, 20E06; Secondary 20E07.

Key words and phrases. Group ring, augmentation ideal of a subgroup, groups acting on trees, fundamental group of a graph of groups, derivation. 
Dunwoody's Theorem. Let $d: G \rightarrow R G$ be a derivation with kernel $H$. If $G$ is finitely generated over $H$ then $G$ acts on a tree $X$

with $X / G$ finite,

with finite edge stabilizers,

with a vertex $v_{0}$ having stabilizer precisely $H$,

such that there exists a right $R G$-linear map $\beta: R[E(X)] \rightarrow R G$ such that $X\left(v_{0}, v_{0} g\right) \beta=(g) d$ for all $g \in G$.

$\$ 2$ below illustrates Theorem $A$ with examples and applications to cases previously considered in the literature.

1. Proof of Theorem A. Let us restate the condition

(1) $\omega(R H) G$ is an $R G$-summand of $\omega(R G)$

as

(4) There is a right $R G$-linear map $\alpha: \omega(R G) \rightarrow \omega(R H) G$ such that $(1-h) \alpha=$ $(1-h)$ for all $h \in H$.

Writing $\pi: R G \rightarrow R[H \backslash G], g \mapsto H g$, we claim that (4) is equivalent to

(5) There is a derivation $d: G \rightarrow R G$ such that $(g) d \pi=H(1-g)$ for all $g \in G$, and $(h) d=0$ for all $h \in H$.

Proof. (4) $\Rightarrow(5)$. Define $(g) d=(1-g)-(1-g) \alpha$.

$(5) \Rightarrow(4)$. Define $(1-g) \alpha=(1-g)-(g) d$ and notice that the kernel of $\pi$ is $\omega(R H) G$, so $\alpha$ has the desired codomain.

Now the kernel of $d$ in (5) is precisely $H$ so assuming $G$ is finitely generated over $H$ we can apply Dunwoody's Theorem to deduce that (5) implies

(6) $G$ acts on a tree $X$

with $X / G$ finite,

with finite edge stabilizers,

with a vertex $v_{0}$ having stabilizer precisely $H$,

such that there exists a right $R G$-linear map $\beta: R[E(X)] \rightarrow R G$ such that $X\left(v_{0}, v_{0} g\right) \beta \pi=H(1-g)$ for all $g \in G$.

Conversely it is easy to see that (6) implies (5) in general since $G \rightarrow R[E(X)]$, $g \mapsto X\left(v_{0}, v_{0} g\right)$, is a derivation with kernel $H$.

Thus it remains to transform (6) into the desired form (3); for convenience let us record what we are proving. (We do not need $G$ finitely generated over $H$ for this step.)

1.1. LEMMA. Let $G$ act on a tree $X$ with $X / G$ finite, with finite edge stabilizers and with a vertex $v_{0}$ having stabilizer precisely $H$. Then the following are equivalent:

$\left(6^{\prime}\right)$ There exists a right $R G$-linear map $\beta: R[E(X)] \rightarrow R G$ such that $X\left(v_{0}, v_{0} g\right) \beta \pi$ $=H(1-g)$ for all $g \in G$.

(3') $\left|H \cap H^{g}\right| \in R^{-1}$ for all $g \notin H$; and for each vertex $v \neq v_{0}$ having star(v) infinite $\left|H \cap G_{v}\right| \in R^{-1}$. 
Proof. $\left(6^{\prime}\right) \Rightarrow\left(3^{\prime}\right)$. Consider any $g \notin H$, and let $x=\left(X\left(v_{0}, v_{0} g\right)\right) \beta \in R G$. Then $x$ is stabilized by $G_{v_{0}} \cap G_{v_{0} g}=H \cap H^{g}$ so the coefficient of $H$ in $x \pi \in R[H \backslash G]$ lies in $R\left|H \cap H^{g}\right|$. But the coefficient of $H$ in $x \pi=H(1-g)$ is 1 so $\left|H \cap H^{8}\right| \in$ $R^{-1}$ as desired.

Now consider any vertex $v \neq v_{0}$ with $\operatorname{star}(v)$ infinite and let $x=\left(X\left(v_{0}, v\right)\right) \beta \in$ $R G$. Then $x$ is stabilized by $G_{v_{0}} \cap G_{v}=H \cap G_{v}$ so the coefficient of $H$ in $x \pi \in R[H \backslash G]$ lies in $R\left|H \cap G_{v}\right|$. But for all $g \in G_{v}$,

$$
x \pi(1-g)=\left(X\left(v_{0}, v\right)(1-g)\right) \beta \pi=\left(X\left(v_{0}, v_{0} g\right)\right) \beta \pi=H(1-g)
$$

so $x \pi-H$ is fixed by $G_{v}$. But $G_{v}$ is infinite since $\operatorname{star}(v) / G_{v} \simeq \operatorname{star}(v G) \subseteq X / G$ is finite. Thus $\left(H \cap G_{v}\right) \backslash G_{v}$ is infinite so the coefficient of $H$ in $x \pi-H$ must be 0; hence the coefficient of $H$ in $x \pi$ is 1 and $\left|H \cap G_{v}\right| \in R^{-1}$ as desired.

$\left(3^{\prime}\right) \Rightarrow\left(6^{\prime}\right)$. Let $T=\left\{x \in X:\left|H \cap G_{x}\right| \notin R^{-1}\right.$ or $\left.x=v_{0}\right\}$. Notice that $T$ is a subtree of $X$ and that $H$ acts on $T$ stabilizing the vertex $v_{0}$, so $T / H$ is also a tree. By $\left(3^{\prime}\right), \operatorname{star}_{X}(v)$ is finite for each $v \in V(T)-\left\{v_{0}\right\}$ so $T / H$ is locally finite (in the sense that every vertex has a finite star). We claim that $T / H$ is finite. Suppose it is not. Then by the König Tree Lemma there is an "infinite path" $\left\{v_{0}\right\}=v_{0} H$, $\left(e_{1} H\right)^{e_{1}},\left(e_{2} H_{2}\right)^{e_{2}}, \ldots$ in $T / H$. This lifts back to an "infinite path" $P: v_{0} e_{1}^{e_{1}}$, $e_{2}^{e_{2}}, \ldots$ in $T$. Now $H \cap G_{e_{1}} \supseteq H \cap G_{e_{2}} \ldots$ and since these are finite groups the chain is eventually constant. By definition of $T$ we can therefore choose an element $h$ that belongs to every term of this chain and whose order is not in $R^{-1}$. Since $X / G$ is finite there is some edge $e$ of $X$ such that $e G$ meets $P$ infinitely often. Say $e g_{1}^{-1}, e g_{2}^{-1}, \ldots$ are distinct edges in $P$. Then $h \in H \cap G_{e g_{i}^{-1}}$ for $i=1,2, \ldots$ so $h^{z_{1}} \in G_{e}$ for $i=1,2, \ldots$ But $G_{e}$ is finite so we must have $h^{g_{1}}=h^{\&}$ for some $i \neq j$. But then $e g_{i}^{-1} H \neq e g_{j}^{-1} H$ so $g_{i} g_{j}^{-1} \notin H$, while $h^{\&}=h^{\&} \in H^{\&} \cap H^{\&}$. This contradicts $\left|H \cap H^{g}\right| \in R^{-1}$ for all $g \notin H$. Hence $T / H$ is finite as claimed.

Recall that the coboundary of $T$ in $X$ is defined as

$$
\delta T=\{e \in E(X) \mid e \text { has one vertex in } T \text { and one vertex not in } T\} .
$$

Since $\delta T \subseteq \cup_{v \in V(T)} \operatorname{star}(v)$, and $\operatorname{star}(v)$ is finite for all $v \in T-\left\{v_{0}\right\}$, we see $\delta T / H$ is finite. Let $E_{0}$ be a (finite) set of representatives in $\delta T$ for the $H$-action. For each $e \in E(X)$ set

$$
r_{e}= \begin{cases}\left|H_{e}\right|^{-1} & \text { if } e \in E_{0} \text { and } \iota \in T, \\ -\left|H_{e}\right|^{-1} & \text { if } e \in E_{0} \text { and } \tau e \in T, \\ 0 & \text { if } e \notin E_{0} .\end{cases}
$$

(Notice that $E_{0} \cap T=\varnothing$ so we may view $r_{e}$ as an element of $R$.) Define $\beta$ : $R[E(X)] \rightarrow R G$ as the $R$-linear map with $e \beta=\Sigma_{g \in G} g r_{e g}{ }^{-1}$. Since almost all the $r_{e}$ are zero, and edge stabilizers are finite, the support of $e \beta$ is finite; clearly $\beta$ is $R G$-linear. The composite $\beta \pi: R[E(X)] \rightarrow R[H \backslash G]$ sends $e$ to $\Sigma_{H g \in H / G} H_{g s}$ eg $^{-1}$ where

$$
s_{e}=\sum_{h \in H} r_{e h^{-1}}=\left|H_{e}\right| \sum_{e h \in e H} r_{e h}=\left\{\begin{aligned}
1 & \text { if } e \in \delta T \text { and } \iota \in T, \\
-1 & \text { if } e \in \delta T \text { and } \tau e \in T, \\
0 & \text { if } e \notin \delta T .
\end{aligned}\right.
$$


Thus for any $g_{0} \notin H$, if $e_{1}^{\varepsilon_{1}}, \ldots, e_{n}^{e_{n}}$ is the geodesic in $X$ from $v_{0}$ to $v_{0} g_{0}$ then $X\left(v_{0}, v_{0} g_{0}\right) \beta \pi=\sum_{H g \in H / G} H g\left(\sum_{i=1}^{n} \varepsilon_{i} s_{e_{i} g^{-1}}\right)$. Now $\left(e_{1} g^{-1}\right)^{\varepsilon_{1}}, \ldots,\left(e_{n} g^{-1}\right)^{\varepsilon_{n}}$ is the geodesic from $v_{0} g^{-1}$ to $v_{0} g_{0} g^{-1}$ so

$$
\begin{aligned}
\sum_{i=1}^{n} \varepsilon_{i} s_{e_{i} g^{-1}} & \left\{\begin{array}{cl}
+1 & \text { if } v_{0} g^{-1} \in T \text { and } v_{0} g_{0} g^{-1} \notin T, \\
-1 & \text { if } v_{0} g^{-1} \notin T \text { and } v_{0} g_{0} g^{-1} \in T, \\
0 & \text { otherwise, }
\end{array}\right. \\
& =\left\{\begin{array}{cl}
+1 & \text { if } g \in H \text { and } g_{0} g^{-1} \notin H, \\
-1 & \text { if } g \notin H \text { and } g_{0} g^{-1} \in H \quad \text { by }\left(3^{\prime}\right), \\
0 & \text { otherwise, }
\end{array}\right. \\
& =\left\{\begin{array}{cl}
+1 & \text { if } H g=H, \\
-1 & \text { if } H g=H g_{0} \\
0 & \text { otherwise. }
\end{array}\right.
\end{aligned}
$$

Hence $X\left(v_{0}, v_{0} g_{0}\right) \beta \pi=H\left(1-g_{0}\right)$ as desired.

(The proof shows that $\left(6^{\prime}\right)$ and $\left(3^{\prime}\right)$ are further equivalent to $\left|H \cap H^{g}\right| \in R^{-1}$ for all $g \notin H$, and $\delta T / H$ is finite.)

In (3), for any vertex $v$ of $X$ consider the action of $G_{v}$ on $\operatorname{star}(v)$. The kernel of the action is finite since dge stabilizers are finite, and there are only finitely many orbits, since $X / G$ is finite. Thus $\operatorname{star}(v)$ is infinite if and only if $G_{v}$ is infinite.

Now applying the Bass-Serre Structure Theorem for groups acting on trees $[[7, \mathrm{p}$. 76], cf. [2, p. 21]) we see that (2) and (3) are equivalent.

This completes the proof of Theorem A.

1.2. REMARK. In Theorem A the rôle played by the condition

$$
\left|H \cap H^{g}\right| \in R^{-1} \quad \text { for all } g \notin H
$$

is to make $\omega(R H) G$ an $R H$-summand of $\omega(R G)$. In fact for any group $G$ the following are equivalent:

(a) $\omega(R H) G$ is an $R H$-summand of $\omega(R G)$.

(b) $\left|H \cap H^{g}\right| \in R^{-1}$ for all $g \notin H$.

(c) $\omega(R G) / \omega(R H) G$ is $R H$-projective.

Proof. (a) $\Rightarrow($ b). Let $\alpha: \omega(R G) \rightarrow \omega(R H) G$ be a splitting. Consider any $g \notin H$ and let $x=(1-g)-(1-g) \alpha$. For $h \in H \cap H^{g}$, say $g h=h^{\prime} g,(1-g)(1-h)$ $=(1-h)-\left(1-h^{\prime}\right) g$ is fixed by $\alpha$, so $x(1-h)=0$. Thus $x$ is fixed by $H \cap H^{g}$ so the coefficient of $H$ in $x \pi$ lies in $R\left|H \cap H^{8}\right|$. But $x \pi=H(1-g)$ and the coefficient of $H$ is 1. So $\left|H \cap H^{g}\right| \in R^{-1}$ as claimed.

(b) $\Rightarrow$ (c). There is an isomorphism of $R H$-modules

$$
\omega(R G) / \omega(R H) G \simeq \underset{H g H \neq H}{\bigoplus} R[H \backslash H(1-g) H] \simeq \underset{H g H \neq H}{\bigoplus} R\left[H \cap H^{g} \backslash H\right]
$$

and the latter is clearly $R H$-projective if (b) holds.

(c) $\Rightarrow(a)$ is clear. 


\section{Examples.}

2.1. ExAmple. Let us illustrate the proof of 1.1 with the simplest case of (2), where $H$ is finite and the graph of groups is just

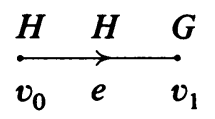

Notice that the fundamental group is $H{ }^{*}{ }_{H} G=G$. The corresponding tree $X$ is

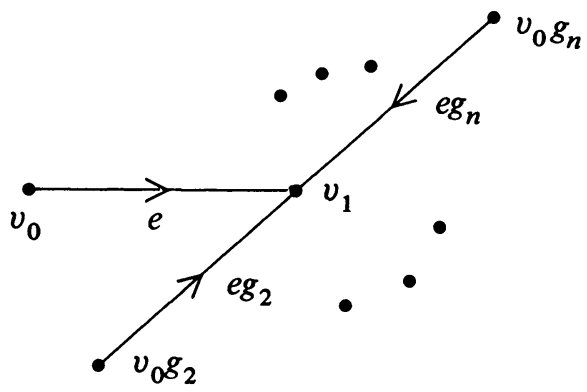

where $H \backslash G=\left\{H, H g_{2}, \ldots, H g_{n}, \ldots\right\}$. There are two possibilities: either $|H| \in$ $R^{-1}$ or $|H| \notin R^{-1}$.

If $|H| \in R^{-1}$ then, in the notation of 1.1,T $=\left\{v_{0}\right\}, \delta T=\{e\}, E_{0}=\{e\}$ and the $\operatorname{map} \beta: R[E(X)] \rightarrow R G$ sends $e$ to $x=|H|^{-1} \Sigma_{h \in H} h$. For each $g \in G, X\left(v_{0}, v_{0} g\right) \beta$ $=(e-e g) \beta=x(1-g)$. The splitting $\alpha: \omega(R G) \rightarrow \omega(R H) G$ is given by left multiplication by the idempotent $1-x \in(R H) G$, and $x \omega(R H)=0$.

If $|H| \notin R^{-1}$ then we impose the condition $\left|H \cap H^{8}\right| \in R^{-1}$ for all $g \notin H$; this implies $T=\left\{v_{0}, e, v_{1}\right\}, \delta T=\left\{e g_{n} \mid n=2,3, \ldots\right\}$ and $E_{0}=\left\{e g_{n_{1}} \mid i=2,3, \ldots\right\}$ where $H \backslash G / H=\left\{H, H g_{n_{2}} H, \ldots, H g_{n_{i}} H, \ldots\right\}$. The condition that $E_{0}$ be finite is the same as $H \backslash G / H$ being finite, i.e. $G$ is finite. The map $\beta: R[E(X)] \rightarrow R G$ sends $e$ to $x=-\Sigma_{i} \mid H \cap H^{g_{n_{i}} \mid \Sigma_{g \in H_{n_{i}}}} g^{-1}$. Then as in the previous case, the splitting $\alpha: \omega(R G) \rightarrow \omega(R H) G$ is given by left multiplication by $1-x$ where $(1-x) \omega(R G) \subseteq \omega(R H) G$, and $x \omega(R H)=0$. (Of course if $H=G$ then $x=0$.)

Let us note two situations where this simplest case of (2) occurs.

2.2. Corollary (COHEN [5, P. 59]). If $G$ is finite then $\omega(R H) G$ is an $R G$-summand of $\omega(R G)$ if and only if $\left|H \cap H^{g}\right| \in R^{-1}$ for all $g \notin H$.

(One direction is given by 1.2 , and the other direction by 2.1.)

2.3. CoRollary. If $\boldsymbol{G}$ is infinite, finitely generated over $\boldsymbol{H}$, and finitarily indecomposable over $H$ then $\omega(R H) G$ is an $R G$-summand of $\omega(R G)$ if and only if either $H=G$ or $|H| \in R^{-1}$.

Proof. If $\omega(R H) G$ is an $R G$-summand of $\omega(R G)$ then there is a finitary decomposition of $G$ over $H$ as in (2), and by the indecomposability of $G$ over $H, G$ must occur as one of the (infinite) vertex groups so either $H=G$ or $|H \cap G| \in$ $R^{-1}$. The converse is clear from 2.1 . 
2.4. EXAMPLE. In the situation of Example 2.1, the conditions in (2) reduced to relatively easily understood restrictions on $H$ and $G$. Let us see what can be said in the next simplest case of (2), where the graph of groups is

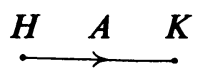

with $A$ finite, so the fundamental group $G$ is the coproduct with amalgamation $H *_{A} K$. Here the conditions (2) state that $\left|H \cap H^{g}\right| \in R^{-1}$, and if $K$ is infinite then $\left|H \cap K^{g}\right| \in R^{-1}$ for all $g \in G$. In particular $A=H \cap K$ and, for $k \in K-$ $A, A \cap A^{k}=(H \cap K) \cap(H \cap K)^{k}=H \cap H^{k}$ so we see

(7) if $K$ is infinite then $|A| \in R^{-1}$, and if $K$ is finite then $\left|A \cap A^{k}\right| \in R^{-1}$ for all $k \in K-A$.

Conversely, (7) suffices, since for any $g \notin H, H \cap H^{8} \subseteq A \cap A^{k}$ for some $k \in K-A$, and for any $g \in G, H \cap K^{g} \subseteq A^{h}$ for some $h \in H$.

Notice that by $2.1,(7)$ implies $\omega(R A) K$ is an $R K$-summand of $\omega(R K)$, so $\omega(R A) G$ is an $R G$-summand of $\omega(R K) G$. Further, Lewin [6] showed that the natural diagram of $R G$-modules

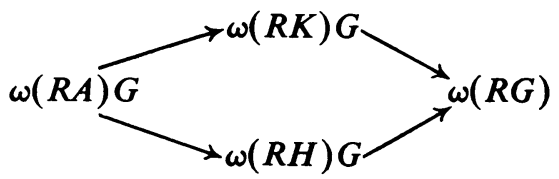

is a pushout and pullback. This makes it clear why $\omega(R H) G$ should be an $R G$-summand of $\omega(R G)$ in this case.

In the special case where $A=1$ and $G=H * K$ we have $\omega(R G)=\omega(R H) G \oplus$ $\omega(R K) G$, which is a very natural way for $\omega(R H) G$ to be an $R G$-summand of $\omega(R G)$. For $H$ torsion-free this is virtually the only way.

2.5. Corollary (Dunwoody [4] AfTer Cohen [1, §7]). Let $G$ be finitely generated over $H$, and let $H$ be torsion-free. Then $\omega(R H) G$ is an $R G$-summand of $\omega(R G)$ if and only if $H$ is a free factor of $G$.

Proof. We have just observed one direction. Conversely, suppose $\omega(R H) G$ is an $R G$-summand of $\omega(R G)$. Then $G$ can be expressed as the fundamental group of a graph of groups $(\mathcal{G}, Y)$ with $Y$ finite, with finite edge groups, and with $H=\mathcal{G}\left(v_{0}\right)$, one of the vertex groups. (This is immediate from Dunwoody's Theorem, and does not require 1.1.) Suppose that $\mathcal{G}$ has been chosen so that $Y$ is as small as possible. It is not difficult to see that each edge of $Y$ has $v_{0}$ as one (or more) of its vertices, so in particular every edge group must be trivial since $H$ has no nontrivial finite subgroups. It follows that $H$ must be a free factor of $G$, as desired.

\section{REFERENCES}

1. D. E. Cohen, Groups of cohomological dimension one, Lecture Notes in Math., vol. 245, SpringerVerlag, Berlin, 1972.

2. W. Dicks, Groups, trees and projective modules, Lecture Notes in Math., vol. 790, Springer-Verlag, Berlin, 1980.

3. M. J. Dunwoody, Accessibility and groups of cohomological dimension one, Proc. London Math. Soc. (3) 38 (1979), 193-215. 
4. __ Recognizing free factors, Homological Group Theory, LMS Lecture Notes No. 36, Cambridge Univ. Press, Cambridge, 1979.

5. K. W. Gruenberg, Relation modules of finite groups, CBMS Regional Conference Series, no. 25, Amer. Math. Soc., Providence, R.I., 1976.

6. J. Lewin, On the intersection of augmentation ideals, J. Algebra 16 (1970), 519-522.

7. J.-P. Serre, Arbres, amalgames et $\mathrm{SL}_{2}$, Astérisque No. 46, Société Math. de France, 1977.

Department of Mathematics, Syracuse University, Syracuse, New York 13210

Current address: Department of Mathematics, Bedford College, London NW1 4NS, England 